\title{
La Edad de Inicio en el Consumo de Drogas, un Indicador de Consumo Problemático
}

\section{The starting Age of Drugs Consumption as an Indicator for Problematic Consumption}

\author{
Teresa Hernández López, Jesús Roldán Fernández, Anunciación Jiménez Frutos, \\ Carlos Mora Rodríguez, Dolores Escarpa Sánchez-Garnica y María Teresa Pérez Álvarez \\ Madrid Salud. Ayuntamiento de Madrid
}

Resumen. En este trabajo se estudia la relación entre el inicio precoz de consumo de tabaco, alcohol y cannabis y los consumos problemáticos, en una muestra de 6280 jóvenes madrileños, entre 15 y 24 años.

La experimentación temprana con tabaco, alcohol (antes de los 14 años) o cannabis (antes de los 15 años) se asocia a prevalencias mas elevadas de consumo diario de tabaco y de cannabis, a patrones de alcohol abusivo en promedio diario y a policonsumo regular o consumo concurrente de 2 o más drogas en el último mes.

También se asocia con consecuencias negativas provocadas por el consumo de alcohol o drogas en el ámbito académico, económico, familiar y social.

Se observa que la fuerza de las asociaciones encontradas aumenta a medida que aumenta el número de sustancias de inicio precoz, resultando esto una constante en la práctica totalidad de las asociaciones estudiadas, lo que sugiere que seria interesante incorporar en estudios futuros el análisis de inicios precoces de consumos agrupados.

Palabras clave: Inicio precoz, tabaco, alcohol, drogas, consumo problemático, adolescencia.

\begin{abstract}
Our research's aim consists on studying the relationship between early beginning of tobacco, alcohol and drugs taking, and problematic consumption, in a sample of 6280 young people from Madrid in a range of 15-24 years old. The early experimentation with tobacco, alcohol (before 14 years old) or cannabis (before 15 years old) is related to a higher prevalence of daily tobacco and cannabis consumption, to a daily alcohol-abusive patterns and to either regular multiple-drugs consumption or concurrent consumption of 2 or more drugs in the last month. It has been found too an association with negative consequences caused by alcohol and drugs consumption in economic, academic, and family life. This research reveals that the strength of these kinds of associations increases with the number of consumed substances very early. In fact, that happens in almost all the analyzed associations, and this is the reason of our suggestion of including an analysis of precocious starting of gathered consumptions in future researches.

Key words: early beginning, tobacco, alcohol, drugs, problematic consumption, adolescent.
\end{abstract}

La correspondencia sobre este artículo deberá dirigirse a Teresa Hernández López. Departamento de Evaluación y Calidad. Madrid Salud. c/ Juan Esplandiu 11, bajo. 28007 Madrid. e-mail: hernandezlt@munimadrid.es 


\section{Introducción}

Los consumos de tabaco, alcohol y drogas de comercio ilegal causan una carga importante de la morbimortalidad general (Ezzati et al., 2002). Las muertes causadas por el alcohol, tabaco y otras drogas suponen un componente importante de la mortalidad por todas las causas especialmente la mortalidad prematura (Britton et al., 2003; Rehm, Giesbrecht, Patra y Roerecke, 2006; Single, Robson, Rehm y Xie, 1999) con los consiguientes costes económicos y sociales (Fenoglio, Parel y Kopp, 2003; Rehm et al., 2007). En Estados Unidos se estima que el $70 \%$ de las muertes en población de 10 a 24 años son debidas a accidentes, lesiones, homicidio y suicidio (Eaton et al., 2006). Los consumos de drogas se relacionan frecuentemente con estas causas. A nivel mundial se estima que el $5 \%$ de las muertes en adolescentes y jóvenes de 15 a 29 años son atribuibles al alcohol (Jeringan, 2001). En un reciente estudio de salud de la ciudad de Madrid, se concluye que la mortalidad atribuible al alcohol en el año 2003 fue del 3\% con importantes diferencias según la edad siendo el grupo de 20 a 34 años el de mayor mortalidad atribuible al alcohol con un $16 \%$, los accidentes son la primera causa de mortalidad atribuible al alcohol en este grupo seguida de homicidios y suicidios (Madrid Salud, 2005).

El consumo de drogas en las sociedades occidentales es un fenómeno extendido fundamentalmente entre los jóvenes, especialmente los varones. Sin embargo se observa, de manera consistente en diversos países, la tendencia a reducirse las clásicas diferencias de consumo inter género en las cohortes más jóvenes, estas cohortes también presentan niveles más elevados de consumo de drogas de comercio ilegal (OED, 2005; Madrid Salud, 2006; Degenhardt et al., 2008). En general, el periodo de riesgo de inicio del consumo se concentra en la adolescencia mediana y tardía, se experimenta antes con drogas de comercio legal y más tarde con sustancias de comercio ilegal y con alto grado de transición a patrones de consumo regular en los tres primeros años tras la experimentación (Wittchen et al., 2008). Un estudio que relaciona el consumo de cannabis en seis países europeos constata que la edad de inicio de consumo es menor en los países con más altas prevalencias de consumo de esta sustancia en la población adolescente (Kokkevi, Nic Gabhainn y Spyropoulou, 2006).

La edad de inicio de consumo de las diversas drogas es una variable explorada clásicamente en los estudios de base poblacional sobre consumo, básicamente en los referidos a población adolescente y joven, ya que directamente señala edades diana en las cuales deben implantarse acciones preventivas eficaces tanto de prevención primaria universal como de prevención de trastornos de abuso y dependencia. Actualmente se dispone de mayor evidencia sobre la efectividad de los programas escolares de prevención de consumo de alcohol, tabaco y drogas de comercio ilegal (Fernández, Nebot y Jané, 2002; Faggiano, Vigna-Taglianti, Versino, Zambon, Borraccino y Lemma, 2007; Thomas y Perera 2007) así como de las intervenciones preventivas en el ámbito familiar y comunitario (Foxcroft, Ireland, Lister-Sharp, Lowe y Breen, 2007; Grimshaw y Stanton, 2007; otead y Lancaster, 2007) lo que facilita la planificación de respuestas temporalmente adaptadas y efectivas.

Asimismo, se han identificado numerosos factores de riesgo relacionados con el inicio del consumo de sustancias de comercio legal y de cannabis. Estudios prospectivos, estudios en pares de gemelos y diversas revisiones señalan factores individuales (sexo, edad, rendimiento escolar), familiares (consumo de padres o hermanos, problemática familiar, vigilancia y relación con los padres) factores de influencia social y del entorno como el consumo por parte de los pares y la disponibilidad de sustancias asociados al inicio del consumo (Hayatbakhsh, Mamun, Najman, O'Callaghan, Bor y Alati, 2008; Kendler, Schmitt, Aggen, y Prescott, 2008; Khuder, Price, Jordan, Khuder y Silvestri, 2008; Orwin, 2009). También se asocian, al inicio del consumo, factores como los eventos estresantes en la infancia (Andersen y Teicher, 2008) y el menor nivel socioeconómico en la infancia y adolescencia, aunque este último aspecto resulta más controvertido (Degenhardt et al., 2008; Lemstra et al., 2008; Daniel et al., 2009).

Fowler et al. (2007), en un reciente estudio con pares de gemelos que explora la relación entre el inicio y la progresión a consumo problemático del tabaco, alcohol y cannabis y la influencia de los fac- 
tores ambientales y genéticos, encuentran mayor índice de progresión a consumo más intensivo o regular en tabaco y cannabis que en alcohol. Destacan los factores ambientales con más peso en la iniciación del consumo de alcohol que los genéticos, y estos explicarían en gran parte el consumo intensivo en el alcohol. En tabaco y cannabis los factores genéticos y ambientales son responsables en proporciones similares de la iniciación y de la transición a consumos problemáticos $\mathrm{y}$, aunque con cautelas, concluye que las intervenciones dirigidas a evitar el inicio precoz estarían indicadas en tabaco y cannabis mientras que en el alcohol las investigaciones/acciones deberían enfocarse a identificar y actuar sobre los factores que subyacen al consumo más intensivo o problemático.

El inicio temprano de consumo de drogas se encuentra asociado a consumos problemáticos y a otros comportamientos de riesgo. El consumo precoz de tabaco se ha visto asociado a consumo habitual en la edad adulta y a menor interés y confianza en las capacidades para dejar de fumar, también a inicio de consumo de alcohol y consumo problemático de alcohol (Lando et al., 1999; Mathers, Toumbourou, Catalano, Williams y Patton, 2006). Un estudio que evalúa la edad de inicio de tabaco en mujeres gestantes y el abandono del mismo en el embarazo, encuentra que la iniciación antes de los 15 años se asocia a menor abstinencia en la gestación (Chen, Stanton, Shankaran y Li, 2006).

El inicio precoz de alcohol también se ha visto asociado con consumo de alcohol más intensivo, con más frecuencia de borracheras, con consumos más prevalentes de otras drogas (Vieira, Ribeiro y Laranjeira, 2007) y también con trastornos de abuso/dependencia del alcohol en la edad adulta (Grant et al., 2006; Warner, White y Johnson, 2007).

Un estudio longitudinal concluye que los comienzos tempranos en el consumo de tabaco, alcohol y cannabis se relacionan con más altos niveles de transición a trastornos de abuso y dependencia, sobretodo en el tabaco, sin embargo la transición mas rápida a abuso se observó para el cannabis (Behrendt, Wittchen, Höfler, Lieb y Beesdo, 2009).

DuRant et al. (1999) analizaron la relación entre consumo precoz (edad de 11 años o menos) de drogas y comportamientos de riesgo para la salud (incluidos indicadores de violencia, de suicidio, viajar con un conductor bajo efectos de alcohol o no llevar casco en motocicleta) en estudiantes adolescentes, y encontraron para el consumo precoz de tabaco la correlación mas fuerte con los comportamientos problemáticos, también los consumos precoces de alcohol, cannabis y cocaína se asociaron a una agrupación de conductas de riesgo.

Una reciente revisión sobre cannabis concluye que el inicio precoz de consumo de esta sustancia y su uso regular en la adolescencia se asocian con consumo problemático de cannabis y de otras drogas, con menor rendimiento escolar, deterioro de la salud mental, conductas sexuales de riesgo y delincuencia (Copeland y Swift, 2009). También estudiando parejas de gemelos se encuentra el consumo precoz de cannabis asociado a mayor riesgo de consumo de drogas de comercio ilegal, y problemas de abuso y/o dependencia (Lynskey et al., 2003; Agrawal, Neale, Prescott y Kendler, 2004), otros estudios sobre cannabis precoz encuentran asociación con bajo rendimiento escolar (Hall, 2006). El consumo de cannabis constituye un factor de riesgo para el desarrollo de psicosis, apreciándose una relación temporal y de dosis respuesta, además se confirma la importante influencia del consumo precoz para el desarrollo de síntomas psicóticos (Arseneault, Cannon, Poulton, Murray, Caspi y Moffitt, 2002; Hall, 2006; Roncero, Collazos, Valero y Casas, 2007; Stefanis, Delespaul, Herquet, Bakoula, Stefanis y van Os, 2004).

Investigaciones sobre inicio de consumo de otras drogas ilegales, encuentran asociaciones similares a las descritas, una realizada sobre el consumo de inhalables volátiles (sustancias de iniciación para adolescentes en determinados contextos sociales) encuentra asociación entre consumir estas sustancias antes de los 14 años con el consumo posterior de heroína (Storr, Westergaard y Anthony, 2005).

Según lo anteriormente expuesto, el inicio precoz del consumo de sustancias se considera uno de los mejores predictores de trastornos de abuso o dependencia; sin embargo, son escasas las investigaciones que analizan el inicio precoz de las drogas de mayor prevalencia de consumo (alcohol, tabaco y cannabis) de manera agrupada y las implicaciones de dicha agrupación. En este sentido, Agrawal (2006) llevó a cabo un estudio con mujeres en el que encon- 
tró asociación entre el consumo precoz de tabaco, alcohol y cannabis con experimentar con drogas de comercio ilegal, señalando que el inicio precoz en más de una sustancia contribuyó a aumentar el riesgo de experimentar con otras drogas.

Las realidades locales dependiendo de la extensión de los consumos de drogas en la adolescencia, de sus edades de inicio, y de factores como la permisividad social respecto a algunos consumos, pueden presentar diferencias importantes y se requieren estudios locales que aporten la información necesaria respecto de estas cuestiones, para la mejor planificación preventiva.

El objetivo del presente trabajo es estudiar la relación entre el inicio precoz del consumo de alcohol, tabaco y cannabis y los consumos problemáticos en la población adolescente y joven de la ciudad de Madrid. La base de datos de la encuesta 2005 sobre consumo de drogas en población de 12 a 24 años del Ayuntamiento de Madrid permite una aproximación a estos aspectos desde la perspectiva de género y edad.

\section{Objetivo}

Conocer la edad de inicio de consumo de las diferentes sustancias y su asociación con consumos problemáticos.

\section{Material y método}

\section{Tipo de diseño}

\section{Estudio trasversal.}

\section{Ámbito de estudio}

Universo: Población de 12 a 24 años del municipio de Madrid.

Muestra: Se realizo un muestreo polietápico con afijación disproporcional por edad y distrito -con el objeto de dotar de representatividad a cada uno de los 21 distritos de Madrid- con un tamaño de 7500 entrevistas con un margen de error $\pm 1,13 \%$, con un grado de confianza del $95,5 \% \mathrm{p}=\mathrm{q}=0,5$.

\section{Variables:}

- Sociodemográficas: edad y sexo.

- Consumo de Drogas:

Sustancias: Tabaco, alcohol, cannabis, cocaína, éxtasis y drogas de diseño, anfetaminas, alucinógenos, inhalables volátiles, heroína y tranquilizantes (sin especificar si son o no prescritos).

Para todas las sustancias se pregunta por el consumo alguna vez en la vida, consumo en los últimos 12 meses para las sustancias de comercio ilegal, consumo en el último mes para todas las sustancias incluyendo diferentes frecuencias de consumo.

- Edad de inicio de consumo para cada droga.

- Consecuencias del consumo de alcohol o drogas:

Faltar a clase, bajar el rendimiento en los estudios, conflictos familiares, problemas en el trabajo, problemas económicos, conflictos con los amigos, enfermedades y otros. Faltar al trabajo sólo para mayores de 18 años.

- Circunstancias relacionadas con el consumo de alcohol y/o drogas:

Sufrir accidentes después de consumir alcohol o drogas, involucrarse en peleas o discusiones tras consumo de alcohol o drogas, o haber conducido vehículos después de tomar alcohol o drogas (sólo para mayores de 18 años).

- Inicio precoz de tabaco, alcohol y cannabis: Para definir el corte de la "edad de inicio precoz" se ha considerado la edad de inicio que se situara entre el percentil 30-35 en cada una de las sustancias. Resultando para el tabaco y el alcohol el inicio precoz el realizado antes de los 14 años y en el caso del cannabis la iniciación antes de los 15 años.

- Bebedor abusivo o de riesgo: consumo de alcohol en promedio diario $\geq 50 \mathrm{cc}$ en varones y $\geq 30 \mathrm{cc}$ en mujeres.

- Policonsumo regular en el último mes. Se establecen los criterios de consumo concurrente de dos o más sustancias en los 30 días previos a la encuesta. Considerando para el tabaco el consumo diario, para el alcohol presentar patrón de bebedor abusivo, y para todas las sustancias de comercio ilegal y los tranquilizantes una fre- 
cuencia de consumo de 2 o más días por semana en el último mes.

\section{Recogida y análisis de datos}

Se han obtenido de la base de datos de la encuesta 2005 a población adolescente y joven (12-24 años) de la ciudad de Madrid. Para el presente estudio se seleccionó la población de 15 a 24 años (n: 6280).

La media y desviación estándar describen las variables cuantitativas. Para las variables cualitativas se presenta la estimación puntual y el intervalo, con un nivel de confianza del $95 \%$. El análisis se ha realizado mediante el paquete estadístico SPSS.

\section{Resultados}

El consumo de drogas está ampliamente extendido entre los adolescentes y jóvenes madrileños. Las sustancias de comercio legal como el alcohol y el tabaco presentan las prevalencias más elevadas, la experimentación con alcohol la refiere el 92,5\% de la población y el $63,7 \%$ con el tabaco. El cannabis es la sustancia de comercio ilegal mas consumida por los jóvenes madrileños, el 59\% la ha probado en alguna ocasión. Un 94\% de los jóvenes han probado una o más de estas tres sustancias.

La iniciación al consumo de drogas de comercio legal en Madrid es temprana en la actualidad. El tabaco y el alcohol presentan las edades de inicio más precoces -tabaco 14,1 años de media $(\mathrm{Sx}=2,39)$, alcohol 14,3 $(\mathrm{Sx}=2,08)$.

Los inhalables y el cannabis son las drogas de comercio ilegal de iniciación de los jóvenes madrileños $-15,4$ años edad media $(S x=2,68)$ para inhalables y $15,6(\mathrm{Sx}=2,15)$ para el cannabis-. Para el resto de drogas los 17 años concentran las edades medias. No se observan diferencias inter género en la edad media de inicio de las diferentes sustancias (Ver Grafico 1)

El $42 \%$ de la población que ha experimentado alguna vez con tabaco, alcohol o cannabis presenta "inicio precoz" de consumo de alguna de estas sus-

Gráfico 1. Edad media de inicio de consumo de las diversas drogas según sexo

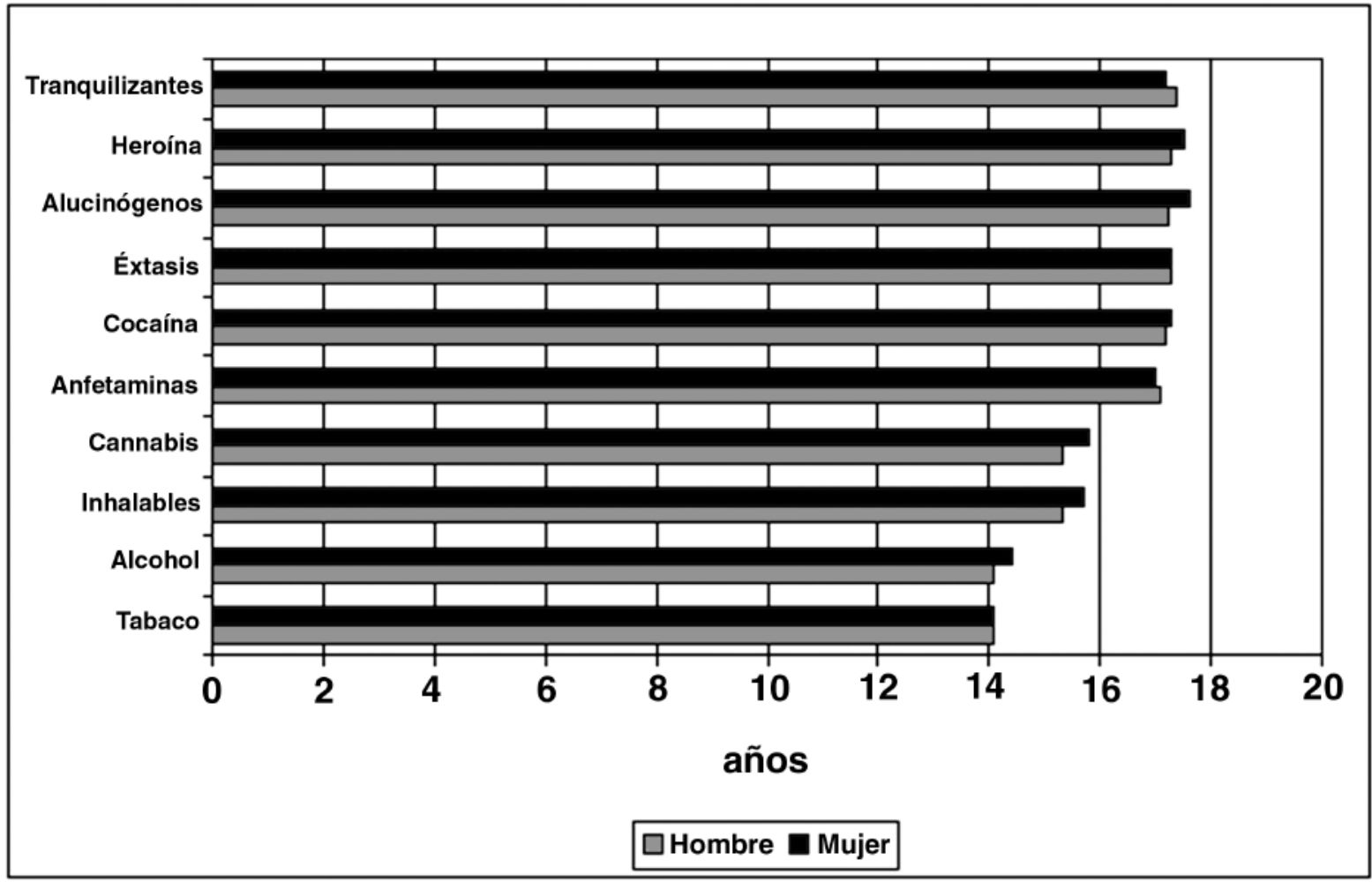


tancias, según los criterios establecidos para este estudio. El inicio precoz se da en mayor proporción en varones que en mujeres $(45,1 \% \mathrm{H}$ y $38,7 \% \mathrm{M})$. Se observan diferencias significativas inter género en el inicio precoz de alcohol (H 32,3\% y M 26,4\%) y cannabis (H 22,8\% y M 15\%) y no se observan diferencias en el inicio precoz de consumo de tabaco.

El inicio precoz del consumo de tabaco, alcohol o cannabis se asocia con la edad. Los más jóvenes pre- alcohol o cannabis se asocia a prevalencias mas elevadas de consumo reciente (últimos 12 meses) de todas las drogas de comercio ilegal, a excepción de la heroína (Ver Gráficos 3 y 4, y Tabla 2). También se asocia a mayor prevalencia de consumo diario de tabaco y de cannabis y a patrones de alcohol abusivo en promedio diario. La asociación es más fuerte a medida que aumenta el número de sustancias de inicio precoz (Ver Grafico 3 y Tabla 2).

\section{Gráfico 2. Distribución porcentual del inicio precoz de consumo de una, dos y tres drogas según sexo y edad}

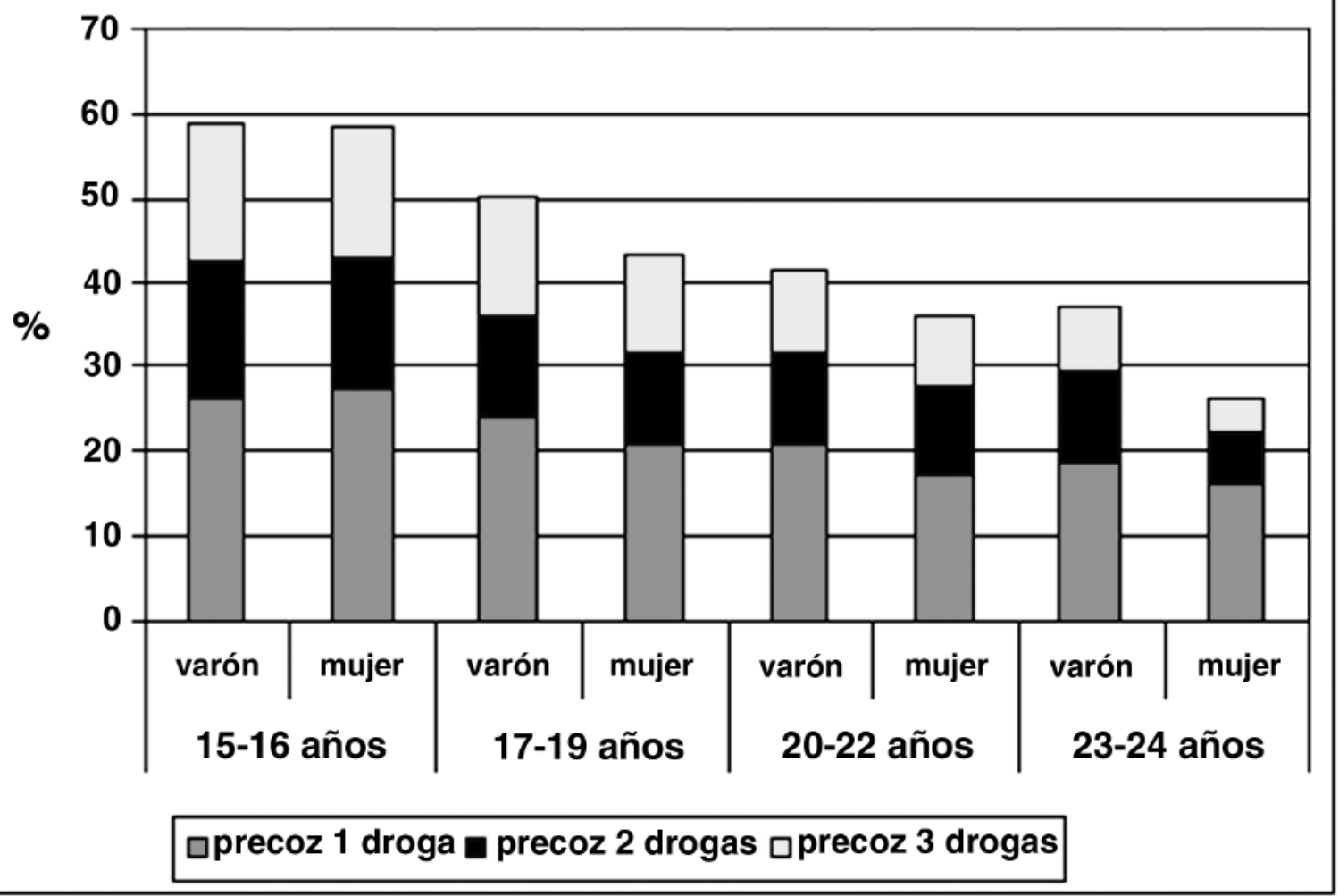

sentan en mayor proporción inicio precoz de consumo que los de más edad, tanto en varones como en mujeres el inicio precoz de una o más sustancias disminuye significativamente a medida que se aumenta la edad (Ver Gráfico 2 y Tabla 1).

Los patrones de inicio precoz de una, dos y tres drogas en los mas jóvenes son iguales para ambos sexos, a medida que aumentamos en edad los patrones se diferencian observándose diferencias significativas entre varones y mujeres de 23-24 años en el consumo precoz de dos y de tres drogas (Ver Tabla 1).

Haber experimentado precozmente con tabaco
El policonsumo regular o consumo concurrente de 2 o más drogas en el último mes (tabaco diario, alcohol abusivo y consumo de 2 o mas días por semana en el resto de sustancias) también se asocia de manera significativa con haber consumido precozmente alguna de las tres drogas y así mismo la fuerza de la asociación se incrementa a medida que aumenta el número de drogas de inicio precoz, resultando que más de la mitad $(55,7 \%)$ de los experimentadores precoces de las tres drogas lo presenta (Ver Tabla 2).

Las asociaciones antes señaladas de consumo 
Tabla 1. Distribución porcentual del inicio (precoz y no precoz) de una, dos y tres sustancias* según edad y sexo

\begin{tabular}{|c|c|c|c|c|c|c|c|c|c|c|}
\hline & \multicolumn{2}{|c|}{$\mathbf{N}^{* * *}$} & \multicolumn{2}{|c|}{$\begin{array}{c}\text { Inicio } \\
\text { no precoz }\end{array}$} & \multicolumn{2}{|c|}{$\begin{array}{c}\text { Inicio precoz } \\
1 \text { droga }\end{array}$} & \multicolumn{2}{|c|}{$\begin{array}{l}\text { Inicio precoz } \\
2 \text { drogas }\end{array}$} & \multicolumn{2}{|c|}{$\begin{array}{l}\text { Inicicio precoz } \\
3 \text { drogas }\end{array}$} \\
\hline & Varon & Mujer & Varón & Mujer & Varon & Mujer & Varon & Mujer & Varon & Mujer \\
\hline 15-16 años & 357 & 387 & $\begin{array}{c}41,2 \\
( \pm 5,1)^{* *}\end{array}$ & $\begin{array}{c}41,6 \\
( \pm 4,9)\end{array}$ & $\begin{array}{c}26,1 \\
( \pm 4,6)\end{array}$ & $\begin{array}{c}27,6 \\
( \pm 4,5)\end{array}$ & $\begin{array}{c}16,5 \\
( \pm 3,9)\end{array}$ & $\begin{array}{c}15,2 \\
( \pm 3,6)\end{array}$ & $\begin{array}{c}16,2 \\
( \pm 3,8)\end{array}$ & $\begin{array}{c}15,5 \\
( \pm 3,6)\end{array}$ \\
\hline 17-19 años & 892 & 885 & $\begin{array}{c}49,9 \\
( \pm 3,3)\end{array}$ & $\begin{array}{c}56,9 \\
( \pm 3,3)\end{array}$ & $\begin{array}{c}24,1 \\
( \pm 2,8)\end{array}$ & $\begin{array}{c}20,8 \\
( \pm 2,7)\end{array}$ & $\begin{array}{c}12,2 \\
( \pm 2,1)\end{array}$ & $\begin{array}{c}11,0 \\
( \pm 2,1)\end{array}$ & $\begin{array}{c}13,8 \\
( \pm 2,3)\end{array}$ & $\begin{array}{c}11,3 \\
( \pm 2,1)\end{array}$ \\
\hline 20-22 años & 1017 & 963 & $\begin{array}{l}58,5 \\
( \pm 3)\end{array}$ & $\begin{array}{l}63,8 \\
( \pm 3)\end{array}$ & $\begin{array}{c}20,8 \\
( \pm 2,5)\end{array}$ & $\begin{array}{c}17,2 \\
( \pm 2,4)\end{array}$ & $\begin{array}{c}11,1 \\
( \pm 1,9)\end{array}$ & $\begin{array}{l}10,7 \\
( \pm 2)\end{array}$ & $\begin{array}{c}9,5 \\
( \pm 1,8)\end{array}$ & $\begin{array}{c}8,3 \\
( \pm 1,7)\end{array}$ \\
\hline 23-24 años & 702 & 709 & $\begin{array}{c}62,8 \\
( \pm 3,6)\end{array}$ & $\begin{array}{c}73,8 \\
( \pm 3,2)\end{array}$ & $\begin{array}{c}18,8 \\
( \pm 2,9)\end{array}$ & $\begin{array}{c}16,1 \\
( \pm 2,7)\end{array}$ & $\begin{array}{c}10,7 \\
( \pm 2,3)\end{array}$ & $\begin{array}{c}6,3 \\
( \pm 1,8)\end{array}$ & $\begin{array}{l}7,7 \\
( \pm 2)\end{array}$ & $\begin{array}{c}3,8 \\
( \pm 1,4)\end{array}$ \\
\hline
\end{tabular}

* Sutancias de inicio precoz: tabaco y alcohol antes de 14 años y cannabis antes de 15 años.

** Nivel de confianza al $95 \%$.

*** La población seleccionada para este análisis son los jóvenes que han consumido alguna vez tabaco, alcohol o cannabis.

Gráfico 3. Prevalencias de consumo de diferentes drogas según inicio (precoz y no precoz) de consumo de una, dos o tres sustancias

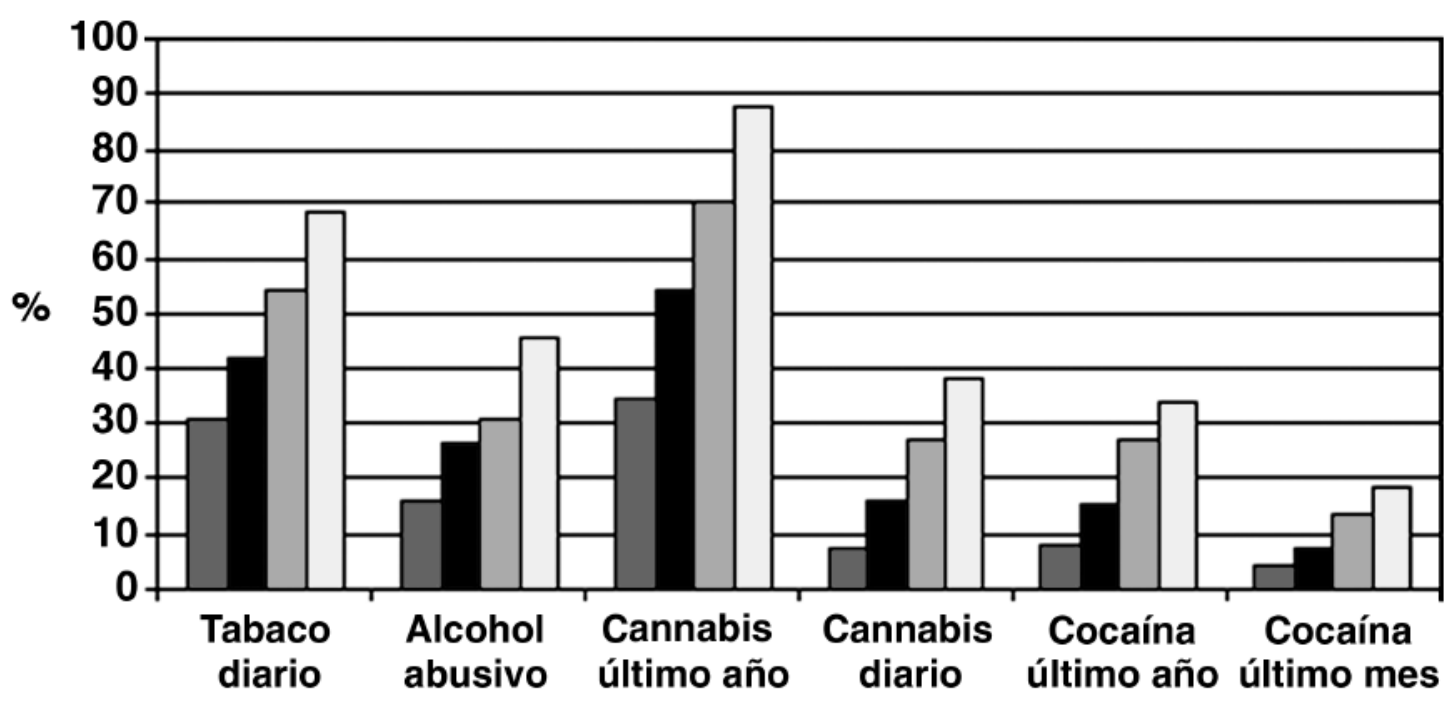

$\square$ inicio no precoz $\square$ precoz 1 droga $\square$ precoz 2 drogas $\square$ precoz 3 drogas

precoz y prevalencias de consumo más elevadas se observan tanto en hombres como en mujeres. Las prevalencias observadas en los jóvenes con algún consumo precoz presentan diferencias entre géneros en el consumo diario de cannabis, en el policonsumo regular en el último mes y en el consumo reciente (últimos 12 meses) de cocaína, cannabis, éxtasis y alucinógenos a favor de los varones. En el consumo reciente de tranquilizantes las diferencias son a favor de las mujeres. No se observan diferencias inter género en los precoces, en las prevalencias de consumo diario de tabaco, el alcohol abusi- 
Gráfico 4. Prevalencias de consumo en el último año según inicio precoz en el consumo de una, dos y tres drogas

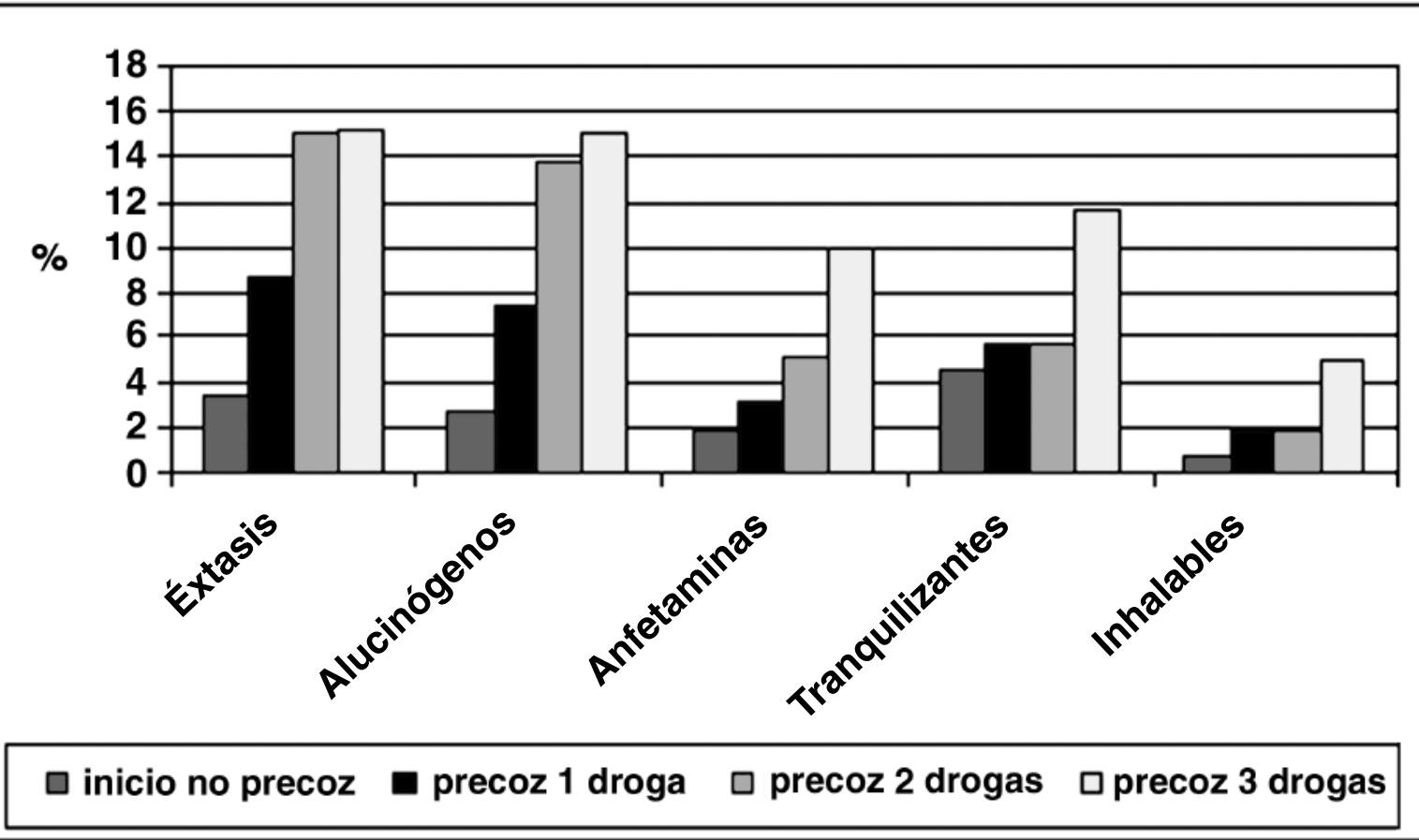

Tabla 2. Prevalencias de consumo de drogas en el último año, último mes y diario, y de alcohol abusivo según inicio (precoz y no precoz) de una, dos o tres sustancias*

\begin{tabular}{|c|c|c|c|c|}
\hline & Inicio no precoz & Inicio precoz de 1 sustancia & Inicio precoz de 2 sustancias & Inicicio precoz de 3 sustancias \\
\hline Tabaco diario & $\begin{array}{c}39,9 \\
( \pm 1,5) * *\end{array}$ & $\begin{array}{c}41,7 \\
( \pm 2,8)\end{array}$ & $\begin{array}{c}53,8 \\
( \pm 3,8)\end{array}$ & $\begin{array}{c}68,3 \\
( \pm 3,7)\end{array}$ \\
\hline Alcohol abusivo & $\begin{array}{c}16 \\
( \pm 1,2)\end{array}$ & $\begin{array}{l}26,3 \\
( \pm 2,5\end{array}$ & $\begin{array}{c}31 \\
( \pm 3,5)\end{array}$ & $\begin{array}{l}45,4 \\
( \pm 4)\end{array}$ \\
\hline Cannabis último año & $\begin{array}{c}34,4 \\
( \pm 1,6)\end{array}$ & $\begin{array}{c}54,1 \\
( \pm 2,8)\end{array}$ & $\begin{array}{c}69,9 \\
( \pm 3,5)\end{array}$ & $\begin{array}{c}87,8 \\
( \pm 2,6)\end{array}$ \\
\hline Cannabis diario & $\begin{array}{c}7,7 \\
( \pm 0,9)\end{array}$ & $\begin{array}{l}15,9 \\
( \pm 2)\end{array}$ & $\begin{array}{c}27,1 \\
( \pm 3,4)\end{array}$ & $\begin{array}{c}38,1 \\
( \pm 3,9)\end{array}$ \\
\hline Cocaína último año & $\begin{array}{c}8,2 \\
( \pm 0,9)\end{array}$ & $\begin{array}{l}14,7 \\
( \pm 2)\end{array}$ & $\begin{array}{c}26,8 \\
( \pm 3,4)\end{array}$ & $\begin{array}{c}33,9 \\
( \pm 3,8)\end{array}$ \\
\hline Cocaína último mes & $\begin{array}{c}4,1 \\
( \pm 0,7)\end{array}$ & $\begin{array}{c}7,2 \\
( \pm 1,4)\end{array}$ & $\begin{array}{c}13,2 \\
( \pm 2,6)\end{array}$ & $\begin{array}{c}18,2 \\
( \pm 3,1)\end{array}$ \\
\hline Éxtais último año & $\begin{array}{c}3,4 \\
( \pm 0,6)\end{array}$ & $\begin{array}{c}8,7 \\
( \pm 1,6)\end{array}$ & $\begin{array}{c}15 \\
( \pm 2,7)\end{array}$ & $\begin{array}{c}15,3 \\
( \pm 2,9)\end{array}$ \\
\hline Anfetaminas último año & $\begin{array}{c}1,9 \\
( \pm 0,5)\end{array}$ & $\begin{array}{l}3,2 \\
( \pm 1)\end{array}$ & $\begin{array}{c}5,1 \\
( \pm 1,7)\end{array}$ & $\begin{array}{c}10 \\
( \pm 2,4)\end{array}$ \\
\hline Alucinógenos último año & $\begin{array}{c}2,8 \\
( \pm 0,5)\end{array}$ & $\begin{array}{c}7,4 \\
( \pm 1,5)\end{array}$ & $\begin{array}{c}13,8 \\
( \pm 2,6)\end{array}$ & $\begin{array}{c}15 \\
( \pm 2,9)\end{array}$ \\
\hline Inhalables último año & $\begin{array}{c}0,8 \\
( \pm 0,3)\end{array}$ & $\begin{array}{c}1,8 \\
( \pm 0,8)\end{array}$ & $\begin{array}{c}1,9 \\
( \pm 1,1)\end{array}$ & $\begin{array}{c}5 \\
( \pm 1,7)\end{array}$ \\
\hline Tranquilizantes último año & $\begin{array}{c}4,6 \\
( \pm 0,7)\end{array}$ & $\begin{array}{c}5,7 \\
( \pm 1,3)\end{array}$ & $\begin{array}{c}5,6 \\
( \pm 1,8)\end{array}$ & $\begin{array}{c}11,7 \\
( \pm 2,6)\end{array}$ \\
\hline Heroína último año & $\begin{array}{c}0,0 \\
( \pm 0,1)\end{array}$ & $\begin{array}{c}0,4 \\
( \pm 0,4)\end{array}$ & $\begin{array}{l}1,6 \\
( \pm 1)\end{array}$ & $\begin{array}{c}0,6 \\
( \pm 0,7)\end{array}$ \\
\hline $\begin{array}{l}\text { Policonsumo regular } \\
\text { último mes }\end{array}$ & $\begin{array}{c}21,3 \\
( \pm 1,4)\end{array}$ & $\begin{array}{c}38,3 \\
( \pm 2,7)\end{array}$ & $\begin{array}{c}54,5 \\
( \pm 3,8)\end{array}$ & $\begin{array}{c}71,4 \\
( \pm 3,6)\end{array}$ \\
\hline $\mathrm{N}^{* * * *}$ & 3.430 & 1.222 & 659 & 600 \\
\hline
\end{tabular}


Tabla 3. Prevalencias de consumo de drogas en el último año, último mes y diario, y consumo abusivo de alcohol según inicio de consumo (precoz y no precoz) de alguna sustancia y sexo*

\begin{tabular}{|c|c|c|c|c|}
\hline & \multicolumn{2}{|c|}{ Inicio no precoz } & \multicolumn{2}{|c|}{ Inicio precoz } \\
\hline & Varón & Mujer & Varón & Mujer \\
\hline Policonsumo regular último mes & $\begin{array}{c}16,3 \\
( \pm 1,8)^{* *}\end{array}$ & $\begin{array}{c}11,2 \\
( \pm 1,5)\end{array}$ & $\begin{array}{c}40,4 \\
( \pm 2,6)\end{array}$ & $\begin{array}{c}32,7 \\
( \pm 2,7)\end{array}$ \\
\hline Cannabis diario & $\begin{array}{c}10,7 \\
( \pm 1,5)\end{array}$ & $\begin{array}{c}5 \\
( \pm 1)\end{array}$ & $\begin{array}{c}30 \\
( \pm 2,5)\end{array}$ & $\begin{array}{c}17,5 \\
( \pm 2,2)\end{array}$ \\
\hline Cannabis último año & $\begin{array}{c}39,6 \\
( \pm 2,4)\end{array}$ & $\begin{array}{c}29,8 \\
( \pm 2,1)\end{array}$ & $\begin{array}{c}70,2 \\
( \pm 2,4)\end{array}$ & $\begin{array}{c}61,9 \\
( \pm 2,2)\end{array}$ \\
\hline Cocaína último año & $\begin{array}{c}11,4 \\
( \pm 1,2)\end{array}$ & $\begin{array}{c}5,4 \\
( \pm 0,8\end{array}$ & $\begin{array}{c}26,3 \\
( \pm 1,9)\end{array}$ & $\begin{array}{c}18,1 \\
( \pm 2,2)\end{array}$ \\
\hline Éxtais último año & $\begin{array}{c}4,2 \\
( \pm 1,2)\end{array}$ & $\begin{array}{c}2,8 \\
( \pm 0,8)\end{array}$ & $\begin{array}{c}15,1 \\
( \pm 1,9)\end{array}$ & $\begin{array}{c}8,2 \\
( \pm 1,6)\end{array}$ \\
\hline Alucinógenos último año & $\begin{array}{c}3,8 \\
( \pm 0,9)\end{array}$ & $\begin{array}{c}1,8 \\
( \pm 0,6)\end{array}$ & $\begin{array}{c}13,8 \\
( \pm 1,8)\end{array}$ & $\begin{array}{c}7,5 \\
( \pm 1,5)\end{array}$ \\
\hline Tranquilizantes último año & $\begin{array}{c}3,7 \\
( \pm 0,9)\end{array}$ & $\begin{array}{c}5,4 \\
( \pm 1)\end{array}$ & $\begin{array}{c}4,8 \\
( \pm 1,1)\end{array}$ & $\begin{array}{c}9,9 \\
( \pm 1,7)\end{array}$ \\
\hline Tabaco diario & $\begin{array}{c}29,9 \\
( \pm 2,2)\end{array}$ & $\begin{array}{c}32 \\
( \pm 2,2)\end{array}$ & $\begin{array}{c}49,2 \\
( \pm 2,7)\end{array}$ & $\begin{array}{c}53,9 \\
( \pm 2,9)\end{array}$ \\
\hline Bebedor abusivo en promedio diario & $\begin{array}{c}17,4 \\
( \pm 0,7)\end{array}$ & $\begin{array}{c}14,8 \\
( \pm 0,6)\end{array}$ & $\begin{array}{c}32,3 \\
( \pm 2,5)\end{array}$ & $\begin{array}{c}32,2 \\
( \pm 2,7)\end{array}$ \\
\hline Anfetaminas último año & $\begin{array}{c}2,3 \\
( \pm 0,7)\end{array}$ & $\begin{array}{c}1,6 \\
( \pm 0,6)\end{array}$ & $\begin{array}{c}5,4 \\
( \pm 1,2)\end{array}$ & $\begin{array}{c}5,3 \\
( \pm 1,3)\end{array}$ \\
\hline Inhalables último año & $\begin{array}{c}1,4 \\
( \pm 0,6) \\
\end{array}$ & $\begin{array}{c}0,4 \\
( \pm 0,3) \\
\end{array}$ & $\begin{array}{c}3 \\
( \pm 0,9) \\
\end{array}$ & $\begin{array}{c}2,2 \\
( \pm 0,9) \\
\end{array}$ \\
\hline Heroína último año & $\begin{array}{c}0,1 \\
( \pm 0,2)\end{array}$ & $\begin{array}{c}0,0 \\
( \pm 0,0)\end{array}$ & $\begin{array}{c}1 \\
( \pm 0,5)\end{array}$ & $\begin{array}{c}0,6 \\
( \pm 0,4)\end{array}$ \\
\hline $\mathbf{N} * * *$ & 1.628 & 1.802 & 1.340 & 1.141 \\
\hline
\end{tabular}

vo en promedio diario, ni en el consumo reciente de inhalables, anfetaminas y heroína. Si comparamos con las diferencias inter género en la población con inicio no precoz en el consumo observamos que son similares a las de la población de inicio precoz, excepto en el consumo reciente de éxtasis y de tranquilizantes en los que no se observaron diferencias entre hombres y mujeres en el segundo grupo (Ver Tabla 3).

Asimismo el inicio precoz del consumo se asocia a presentar con mayor frecuencia consecuencias negativas provocadas por el consumo de alcohol o drogas, consecuencias en el ámbito académico, económico, familiar y social. También la asociación de estas consecuencias con el consumo precoz es más fuerte a medida que aumenta el número de sustancias con inicio precoz (Ver Tabla 4).

No se observan diferencias en cuanto a consecuencias en el ámbito laboral excepto para los que se iniciaron precozmente en el consumo de las tres sustancias. Los jóvenes madrileños presentan con muy baja frecuencia enfermedades provocadas por el consumo tanto si se iniciaron precozmente, o no, en las sustancias investigadas y sin diferencias entre ellos (Ver Tabla 4).

Los jóvenes con inicio precoz presentan en mayor proporción situaciones o circunstancias problemáticas relacionadas con el consumo de drogas como sufrir accidentes (de tráfico y otros menores) tras el consumo, implicarse en discusiones o riñas y conducir bajo los efectos de alcohol o drogas (Ver Tabla 5). 
Tabla 4. Consecuencias que se han producido en varias ocasiones por el consumo de alcohol o drogas. Distribución porcentual según inicio (precoz y no precoz) de una, dos o tres sustancias*

\begin{tabular}{|c|c|c|c|c|}
\hline \multicolumn{2}{|c|}{ Inicio no precoz } & \multirow{2}{*}{$\begin{array}{c}\text { Inicio precoz de } 1 \text { droga } \\
9,8 \\
( \pm 1,8)\end{array}$} & \multirow{2}{*}{$\begin{array}{c}\text { Inicio precoz de } 2 \text { drogas } \\
\begin{array}{c}18,5 \\
( \pm 3)\end{array}\end{array}$} & \multirow{2}{*}{$\begin{array}{c}\text { Inicicio precoz de } 3 \text { drogas } \\
\begin{array}{c}23,9 \\
( \pm 3,4)\end{array}\end{array}$} \\
\hline Faltar a clase & $\begin{array}{c}5,6 \\
( \pm 0,8) * *\end{array}$ & & & \\
\hline Bajo rendimiento en estudios & $\begin{array}{c}3,9 \\
( \pm 0,7)\end{array}$ & $\begin{array}{c}7,7 \\
( \pm 1,6)\end{array}$ & $\begin{array}{c}13,7 \\
( \pm 2,7)\end{array}$ & $\begin{array}{c}23 \\
( \pm 3,4)\end{array}$ \\
\hline Conflictos familiares & $\begin{array}{c}2,3 \\
( \pm 0,6)\end{array}$ & $\begin{array}{c}5 \\
( \pm 1,3)\end{array}$ & $\begin{array}{l}7,4 \\
( \pm 2)\end{array}$ & $\begin{array}{c}17,8 \\
( \pm 3,1)\end{array}$ \\
\hline Conflictos con amigos & $\begin{array}{c}1,8 \\
( \pm 0,5)\end{array}$ & $\begin{array}{c}3,3 \\
( \pm 1,1)\end{array}$ & $\begin{array}{c}6,1 \\
( \pm 1,9)\end{array}$ & $\begin{array}{c}8,8 \\
( \pm 2,3)\end{array}$ \\
\hline Problemas económicos & $\begin{array}{c}2,2 \\
( \pm 0,5)\end{array}$ & $\begin{array}{c}4,6 \\
( \pm 1,2)\end{array}$ & $\begin{array}{c}9,2 \\
( \pm 2,3)\end{array}$ & $\begin{array}{c}15,8 \\
( \pm 2,9)\end{array}$ \\
\hline Faltar al trabajo $* * * *$ & $\begin{array}{c}0,8 \\
( \pm 0,3)\end{array}$ & $\begin{array}{c}0,4 \\
( \pm 0,4)\end{array}$ & $\begin{array}{c}2,1 \\
( \pm 1,1)\end{array}$ & $\begin{array}{c}4 \\
( \pm 1,6)\end{array}$ \\
\hline Problemas laborales $* * * *$ & $\begin{array}{c}0,5 \\
( \pm 0,3)\end{array}$ & $\begin{array}{c}0 \\
( \pm 0,0)\end{array}$ & $\begin{array}{c}0,8 \\
( \pm 0,7)\end{array}$ & $\begin{array}{c}1,2 \\
( \pm 0,9)\end{array}$ \\
\hline Enfermedades & $\begin{array}{c}0,3 \\
( \pm 0,2)\end{array}$ & $\begin{array}{c}0,9 \\
( \pm 0,6)\end{array}$ & $\begin{array}{c}1,1 \\
( \pm 0,8)\end{array}$ & $\begin{array}{c}1,3 \\
( \pm 0,9)\end{array}$ \\
\hline $\mathbf{N} * * *$ & 2.837 & 1.095 & 626 & 599 \\
\hline
\end{tabular}

*Sustancias de inicio precoz: tabaco y alcohol antes de 14 años y cannabis antes de 15 años.

** Nivel de confianza $95 \%$.

*** La población seleccionada para este análisis son los jóvenes que han consumido alguna vez tabaco, alcohol o cannabis.

**** Solo a partir de 18 años y habiendo trabajado. Porcentajes referidos a la población seleccionada para el análisis global.

$\overline{\text { Tabla 5. Distribución porcentual de circunstancias problemáticas relacionadas con el consumo de alco- }}$ hol o drogas según inicio (precoz y no precoz) de una, dos o tres sustancias*

\begin{tabular}{|c|c|c|c|c|}
\hline & Inicio no precoz & Inicio precoz de 1 droga & Inicio precoz de 2 drogas & Inicicio precoz de 3 drogas \\
\hline Accidentes tras consumo & $\begin{array}{c}7,6 \\
( \pm 0,9)^{* *}\end{array}$ & $\begin{array}{c}11,1 \\
( \pm 1,8)\end{array}$ & $\begin{array}{c}17 \\
( \pm 2,9)\end{array}$ & $\begin{array}{c}26,7 \\
( \pm 3,5)\end{array}$ \\
\hline $\begin{array}{l}\text { Conduce bajo efectos } \\
\text { de alcohol o drogas } * * * *\end{array}$ & $\begin{array}{c}5,8 \\
( \pm 0,9)\end{array}$ & $\begin{array}{c}8,6 \\
( \pm 1,8)\end{array}$ & $\begin{array}{l}12,9 \\
( \pm 3)\end{array}$ & $\begin{array}{c}29,4 \\
( \pm 4,4)\end{array}$ \\
\hline $\begin{array}{l}\text { Implicado en } \\
\text { discusiones/riñas }\end{array}$ & $\begin{array}{c}14,7 \\
( \pm 1,2)\end{array}$ & $\begin{array}{l}19,9 \\
( \pm 2,2)\end{array}$ & $\begin{array}{c}22,3 \\
( \pm 3,2)\end{array}$ & $\begin{array}{c}33,5 \\
( \pm 3,8)\end{array}$ \\
\hline N: 5911*** & 3.431 & 1.222 & 658 & 600 \\
\hline
\end{tabular}

\section{Conclusiones}

Partiendo de que las asociaciones observadas en este estudio no implican causalidad, sin duda son de interés para la mejor comprensión y toma de decisiones y sugieren la necesidad de ampliar la investigación en este campo.

Resulta pertinente investigar la coexistencia de 
factores o variables psicosociales de riesgo para el inicio de consumo y la progresión a consumo problemático, que permitan el análisis ajustado a estos factores; para ello, sin duda, serían más adecuados diseños prospectivos de cohortes que estudios trasversales como el presente.

Otra limitación radica en la imposibilidad de aproximarnos a la prevalencia de trastornos inducidos por uso de sustancias, ya que la encuesta no incluye test de cribado (diagnostico presuntivo de abuso o dependencia) para alcohol, tabaco y cannabis, por lo que estudiamos la asociación de inicio precoz con consumo diario en tabaco y cannabis. Resulta complejo el estudio de los consumos de riesgo de alcohol en estudios de base poblacional, aunque suelen utilizarse además del consumo abusivo en promedio diario, variables como la frecuencia de borracheras y el consumo abusivo puntual (binge drinking) y otras conductas de riesgo relacionadas con el alcohol.

El consumo problemático resulta un termino polisémico en actual discusión, la definición de consenso utilizada en Europa no incluye el alcohol ni el tabaco y no se adapta a los policonsumos concurrentes o simultáneos que representan la realidad actual en los adolescentes. Otro término con necesidad de conceptualización es el de policonsumo. A pesar de estas consideraciones, en el presente estudio la variable policonsumo regular en el último mes (consumo concurrente de dos o mas drogas con los criterios de: tabaco diario, alcohol abusivo en promedio diario, y 2 o mas días de consumo por semana para el resto de sustancias) constituiría para este estudio la mejor aproximación a consumo problemático, dadas las limitaciones.

Estudiar la edad de inicio en el consumo y establecer edades consideradas de inicio precoz, no puede realizarse sin atender al contexto geográfico y sociocultural y a las tendencias de consumo locales. Las diferencias observadas en los estudios revisados en la edad de corte que establece el inicio precoz, son muy importantes y van desde los 11 a los 18 años (DuRant et al., 1999; Mathers, Toumbourou, Catalano, Williams y Patton, 2006). Los puntos de corte establecidos para el inicio precoz en el estudio de Madrid se realizaron en base a la realidad local.

Se observa que la fuerza de las asociaciones encontradas aumenta a medida que aumenta el número de sustancias de inicio precoz, resultando esto una constante en la práctica totalidad de las asociaciones estudiadas, lo que sugiere que seria interesante incorporar en estudios futuros el análisis de inicios precoces de consumos agrupados.

\section{Referencias}

Agrawal, A., Grant, J. D., Waldron, M., Duncan, A. E., Scherrer, J. F., Lynskey, M. T., Madden, P. A., Bucholz, K. K. y Heath, A. C. (2006). Risk for initiation of substance use as a function of age of onset of cigarette, alcohol and cannabis use: findings in a Midwestern female twin cohort Prev Med. Aug, 43, 25-8.

Agrawal, A., Neale, M.C., Prescott, C.A. y Kendler, K. S. (2004). A twin study of early cannabis use and subsequent use and abuse/dependence of other illicit drugs. Psychol Med. Oct, 34, 122737.

Andersen, S. L. y Teicher, M. H.. (2009). Desperately driven and no brakes: developmental stress exposure and subsequent risk for substance abuse. Neurosci Biobehav Rev, Apr, 33, 516-24.

Arseneaul, L., Cannon, M., Poulton, R., Murray, R., Caspi, A. y Moffitt, T. E. (2002). Cannabis use in adolescence and risk for adult psychosis: longitudinal prospective study. BMJ, 325 (7374), 12123.

Behrendt, S., Wittchen, H.U., Höfler, M., Lieb, R. y Beesdo, K. (2009). Transitions from first substance use to substance use disorders in adolescence: is early onset associated with a rapid escalation? Drug Alcohol Depend, Jan 1, 99, 68-78. Epub 2008 Sep 2.

Britton, A., Nolte, E., White, I. R., Gronbaek, M., Powles, J., Cavallo, F. y McPherson, K. (2003). A comparison of the alcohol-attributable mortality in four European countries. Eur J Epidemiol, 18, 643-51.

Copeland, J. y Swift, W. (2009). Cannabis use disorder: epidemiology and management. Int Rev Psychiatry, Apr, 21, 96-103.

Chen, X., Stanton, B., Shankaran, S. y Li, X. (2006). Age of smoking onset as a predictor of smoking 
cessation during pregnancy. Am J Health Behav, May-Jun, 30, 247-58.

Daniel, J. Z., Hickman, M., Macleod, J., Wiles, N., Lingford-Hughes, A., Farrell, M., Araya, R., Skapinakis, P., Haynes, J. y Lewis, G. (2009). Is socioeconomic status in early life associated with drug use? A systematic review of the evidence. Drug Alcohol Rev, Mar, 28, 142-53.

Degenhardt, L., Chiu, W. T., Sampson, N., Kessler, R. C., Anthony, J. C., Angermeyer, M., Bruffaerts, R., de Girolamo, G., Gureje, O., Huang, Y., Karam, A., Kostyuchenko, S., Lepine, J.P., Mora, M. E., Neumark, Y., Ormel, J. H., Pinto-Meza, A., Posada-Villa, J., Stein, D. J., Takeshima, T. y Wells, J. E. (2008). Toward a global view of alcohol, tobacco, cannabis, and cocaine use: findings from the WHO World Mental Health Surveys. PLoS Med, Jul 1, 5, 141.

DuRant, R. H., Smith, J. A., Kreiter, S. R. y Krowchuk, D. P. (1999). The relationship between early age of onset of initial substance use and engaging in multiple health risk behaviors among young adolescents. Arch Pediatr Adolesc Med, Mar, 153, 286-91.

Eaton, D. K., Kann, L., Kinchen, S., Ross, J., Hawkins, J., Harris, W. A., Lowry, R., McManus, T., Chyen, D., Shanklin, S., Lim, C., Grunbaum, J. A. y Wechsler, H. (2006). Youth risk behavior surveillance-United States, 2005. MMWR Surveill Summ, Jun 9, 55, 1-108.

Ezzati, M., Lopez, A., Rodgers, A., Vander Hoorn, S., Murray, C. et al. (2002). Selected major risk factors and global and regional burden of disease. Lance, 360, 1347-1360.

Faggiano, F., Vigna-Taglianti, F. D., Versino, E., Zambon, A., Borraccino, A. y Lemma, P. (2007). Prevención del consumo de drogas ilegales en las escuelas. (Revisión Cochrane traducida). En: La Biblioteca Cochrane Plus, número 4, 2007. Oxford, Update Software Ltd. Disponible en: http://www.update-software.com. (Traducida de The Cochrane Library, 2007 Issue 4. Chichester, UK: John Wiley \& Sons, Ltd.).

Fenoglio, P., Parel, V. y Kopp, P. (2003). The social cost of alcohol, tobacco and illicit drugs in France, 1997. Eur Addict Res, Jan, 9, 18-28.

Fernández, S., Nebot, M. y Jané, M. (2002).
Evaluación de la efectividad de los programas escolares de prevención del consumo de tabaco, alcohol y cannabis ¿Qué nos dicen los meta-analisis? Rev Esp Salud Pública, 76, 175-187.

Fowler, T., Lifford, K., Shelton, K., Rice, F., Thapar, A., Neale, M. C., McBride, A. y van den Bree, M. B. (2007). Exploring the relationship between genetic and environmental influences on initiation and progression of substance use. Addiction, Mar, 102, 413-22.

Foxcroft, D. R., Ireland, D., Lister-Sharp, D. J., Lowe, G. y Breen, R. (2007). Prevención primaria para el abuso de alcohol en los jóvenes (Revisión Cochrane traducida). En: La Biblioteca Cochrane Plus, número 1, 2007. Oxford, Update Software Ltd. Disponible en: http://www.updatesoftware.com. (Traducida de The Cochrane Library, 2007 Issue 1. Chichester, UK: John Wiley \& Sons, Ltd.).

Grant, J. D., Scherrer, J. F., Lynskey, M. T., Lyons, M. J., Eisen, S.A., Tsuang, M. T., True, W. R. y Bucholz, K. K. (2006). Adolescent alcohol use is a risk factor for adult alcohol and drug dependence: evidence from a twin design. Psychol Med, Jan, 36, 109-18.

Grimshaw, G. M. y Stanton, A. (2007). Intervenciones para el abandono del hábito de fumar en personas jóvenes (Revisión Cochrane traducida). En: La Biblioteca Cochrane Plus, número 4, 2007. Oxford, Update Software Ltd. Disponible en: http://www.update-software.com. (Traducida de The Cochrane Library, 2007 Issue 4. Chichester, UK: John Wiley \& Sons, Ltd.).

Hall, W. D. (2006) Cannabis use and the mental health of young people. Aust $N Z J$ Psychiatry, Feb, 40 (2), 105-13.

Hayatbakhsh, M. R., Mamun, A. A., Najman, J. M., O'Callaghan, M. J., Bor, W. y Alati, R. (2008). Early childhood predictors of early substance use and substance use disorders: prospective study. Aust N Z J Psychiatry, Aug, 42, 720-31.

Jernigan, D. H. (2001). Global Status Report: Alcohol and Young People. Geneva: World Health Organisation.

Kendler, K. S., Schmitt, E., Aggen, S. H. y Prescott, C. A. (2008). Genetic and environmental influences on alcohol, caffeine, cannabis, and nicotine 
use from early adolescence to middle adulthood Arch Gen Psychiatry, Jun, 65, 674-82.

Kokkevi, A., Nic Gabhainn, S. y Spyropoulou, M. (2006). Risk Behaviour Focus Group of the HBSC. Early initiation of cannabis use: a crossnational European perspective. J Adolesc Health, Nov, 39, 712-9.

Khuder, S. A., Price, J. H., Jordan, T., Khuder, S. S. y Silvestri, K. (2008). Cigarette smoking among adolescents in Northwest Ohio: correlates of prevalence and age at onset. Int J Environ Res Public Health, Dec, 5, 278-89.

Lando, H., Thai, D. T., Murray, D. M., Robinson, L. A., Jeffery, R. W., Sherwood, N. E. y Hennrikus, D. J. (1999). Age of initiation, smoking patterns, and risk in a population of working adults. Prev Med, Dec, 29, 590-8.

Lemstra, M., Bennett, N. R., Neudorf, C., Kunst, A., Nannapaneni, U., Warren, L. M., Kershaw, T. y Scott, C. R. (2008). A meta-analysis of marijuana and alcohol use by socio-economic status in adolescents aged 10-15 years. Can J Public Health, May-Jun, 99 (3), 172-7.

Lynskey, M. T., Heath, A. C., Bucholz, K. K., Slutske, W. S., Madden, P. A., Nelson, E. C., Statham, D. J. y Martin, L. (2003). Escalation of drug use in early-onset cannabis users vs co-twin controls. JAMA, 289, 427-33.

Madrid Salud (2005) Instituto de Salud Pública. Estudio de salud de la ciudad de Madrid. Mortalidad atribuible 248- 53. http: //www.ma dridsalud.es.

Madrid Salud (2006). Instituto de Adicciones. Consumo de drogas, percepciones y actuaciones de prevención entre la población adolescente y joven de la Ciudad de Madrid. Disponible en http://www.madridsalud.es/adicciones/otros/CO NSUMO\%20DE\%20DROGAS.pdf

Mathers, M., Toumbourou, J. W., Catalano, R. F., Williams, J. y Patton, G. C. (2006). Consequences of youth tobacco use: a review of prospective behavioural studies. Addiction, Jul, 101, 94858.

Observatorio Español sobre Drogas (OED) (2005). Informe 2004. Situación y tendencias de los problemas de drogas en España. (105-128). Ed. Ministerio de Sanidad y Consumo. Delegación del Gobierno para el Plan Nacional sobre Drogas. Rehm, J., Giesbrecht, N., Patra, J. y Roerecke, M. (2006) Estimating chronic disease deaths and hospitalizations due to alcohol use in Canada in 2002: implications for policy and prevention strategies. Prev Chronic Dis, Oct, 3, A121. Epub 2006 Sep 15.

Rehm, J., Gnam, W., Popova, S., Baliunas, D., Brochu, S., Fischer, B., Patra, J., SarnocinskaHart, A. y Taylor, B. (2007). The costs of alcohol, illegal drugs, and tobacco in Canada, 2002. J Stud Alcohol Drugs, Nov, 68, 886-95.

Roncero, C., Collazos, F., Valero, S. y Casas, M. (2007). Cannabis consumption and development of psychosis: state of the art. Actas Esp Psiquiatr, May-Jun, 5 (3), 182-9.

Single, E., Robson, L., Rehm, J. y Xie, X. (1999). Morbidity and mortality attributable to alcohol, tobacco, and illicit drug use in Canada. Am J Public Health, Mar, 89, 385-90.

Otead, L. F. y Lancaster, T. (2007). Intervenciones para la prevención de la venta de tabaco a menores (Revisión Cochrane traducida). En: La Biblioteca Cochrane Plus, número 4, 2007. Oxford, Update Software Ltd. Disponible en: http://www.update-software.com. (Traducida de The Cochrane Library, 2007 Issue 4. Chichester, UK: John Wiley \& Sons, Ltd.).

Stefanis, N. C., Delespaul, P., Herquet, C., Bakoula, C., Stefanis, C. N. y van Os, J. (2004). Eraly adolescent cannabis exposure and positive and negative dimensions of psicosis. Adiction, 99, 1333-41.

Storr, C. L., Westergaard, R. y Anthony, J. C. (2005). Early onset inhalant use and risk for opiate initiation by young adulthood. Drug Alcohol Depend, Jun 1, 78, 253-61.

Tang, Z. y Orwin, R. G. (2009). Marijuana initiation among American youth and its risks as dynamic processes: prospective findings from a national longitudinal study. Subst Use Misuse, 44, 195211.

Thomas, R. y Perera, R. (2007). Programas escolares para la prevención del hábito de fumar (Revisión Cochrane traducida). En: La Biblioteca Cochrane Plus, número 4, 2007. Oxford, Update Software Ltd. Disponible en: http://www.updatesoftware.com. (Traducida de The Cochrane 
Library, 2007 Issue 4. Chichester, UK: John Wiley \& Sons, Ltd.).

Vieira, D. L., Ribeiro, M. y Laranjeira, R. (2007).

Evidence of association between early alcohol use and risk of later problems. Rev Bras Psiquiatr, Sep, 29, 222-7.

Warner, L. A., White, H. R. y Johnson, V. (2007). Alcohol initiation experiences and family history of alcoholism as predictors of problem-drink- ing trajectories. J Stud Alcohol Drugs, Jan, 68, 56-65.

Wittchen, H. U., Behrendt, S., Höfler, M., Perkonigg, A., Lieb, R., Bühringer, G. y Beesdo, K. (2008). What are the high risk periods for incident substance use and transitions to abuse and dependence? Implications for early intervention and prevention. Int J Methods Psychiatr Res, Jun, 17, S16-29.

Manuscrito recibido: 22/09/2009

Revisión recibida: 06/10/2009

Manuscrito aceptado: 08/10/2009 